\title{
Vom gegenseitigen Nutzen von Historischer Sprachwissenschaft und Sprachtypologie - am Beispiel der Phonologie, der Morphologie und der Pragmatik
}

\section{Wohin sollte die Historische Sprachwissenschaft auch steuern?}

Die Historische Sprachwissenschaft war (allzu) lange Zeit ein Ableger der Mediävistik und weniger der Linguistik. Entsprechend bestand (und besteht immer noch) an vielen Universitäten das primäre Ziel der Historischen Sprachwissenschaft darin, den Studierenden die Kompetenz für die Übersetzung ahd. und mhd. Texte zu vermitteln. Hinzu kam (kommt) das relativ zusammenhangslose Auswendiglernen eines ganzen Katalogs an Lautgesetzen sowie einiger Flexionsregeln - auch dies primär zu Zwecken der richtigen Übersetzung. Am Ende einer solchen Ausbildung bestand die Herausforderung schriftlicher und mündlicher Examensprüfungen darin, ältere Textpassagen zu übersetzen sowie ein paar Gesetze aus dem Katalog der Laut- und Flexionsregeln auf ausgewählte Wortbeispiele anzuwenden, Langvokale zu identifizieren und die eine oder andere Ablautreihe zu bestimmen. Warum-Fragen wurden kaum gestellt.

Das im Kontext des Übersetzens aufkeimende Interesse etwa an Gesetzen morphologischen oder semantischen Wandels (unterschiedliche Wortbedeutungen sind ja allgegenwärtig) wurde mit wenigen Sätzen bedient, doch selten zu einem eigenen Thema gemacht. Das Gros der sprachhistorischen Einführungen - selbst solche neuesten Datums - verharrt ungerührt in diesem Stadium althergebrachter Gemütlichkeit. Unmittelbar erkennbar ist dies an der traditionellen Einteilung in ,Das Althochdeutsche', das Mittelhochdeutsche' und ,das Frühneuhochdeutsche'. Immer noch arbeitet man sich durch diese hermetischen Blöcke, bei denen die thematischen Fäden immer wieder abreißen, um 100 Seiten später - wenn überhaupt - wieder aufgenommen zu werden. Phänomenbezogen 
Denkende wurden (und werden) systematisch entmutigt, sich mit der Entwicklung solcher ,Fäden', z. B. des Umlauts, der Entstehung und Entwicklung des Ablautsystems, der Verschriftung etc., zu befassen und womöglich nach der Ratio dahinter zu fragen. Dabei liefert die schriftlich bezeugte Geschichte der deutschen Sprache den unschätzbaren Vorteil beträchtlicher diachroner Tiefe: 1200 Jahre (vergleichsweise) reich belegter Sprachzeugnisse bilden eine solide Basis für Einsichten in die Prinzipien des Sprachwandels und seine bedingenden Faktoren.

Indessen hat sich mittlerweile einiges verändert: Endlich sind Projekte zur Aufbereitung, Verfügbarmachung und Annotierung historischer Korpora gestartet, die bald präzise und repräsentativ(er)e Aussagen zu sprachgeschichtlichen Abläufen erlauben und so manches bisherige Wissen korrigieren werden. Nichtannotierte Korpora sind schon seit längerer Zeit digital verfügbar (vgl. das Projekt Titus an der Universität Frankfurt: http://titus.uni-frankfurt.de). ,Regionale Sprachgeschichtsforschung' ist ein weiteres Stichwort der konsequenten Umsetzung dessen, was man zwar wusste, doch zu wenig berücksichtigt hat: Die diatopische Vielfalt des Deutschen seit Beginn seiner Überlieferung und die schon frühe Eigenständigkeit der Dialekte. Weitere Differenzierungen werden nun diachron untersucht, z. B. Soziolekte, Fachsprachen, externe wie interne Sprachkontakte. Die Mündlichkeits-/Schriftlichkeitsforschung wird auf die Diachronie ausgeweitet. Auch wurden weitere, bisher stark vernachlässigte sprachliche Domänen (neben der Phonologie und Morphologie) diachron erschlossen, was neuere Untersuchungen zur Wortbildung, zur Syntax, zur Pragmatik, zum graphematischen Wandel zeigen.

Den m. E. entscheidenden Impuls für den Anschluss der Historischen Sprachwissenschaft an linguistische Fragestellungen und Theorien hat die Grammatikalisierungsforschung seit den 1980er Jahren erbracht. Seitdem erfährt die Sprachgeschichte ein bisher nicht dagewesenes Interesse verschiedener linguistischer Disziplinen. Deutlich wurde dabei aber auch: Es bedarf umfassender Kenntnisse sowohl der sprachhistorischen Daten als auch der Theorie(n). Das eine schließt also das andere nicht aus, beides ergänzt sich und ist gleichermaßen erforderlich, um echte Erkenntnisfortschritte zu erzielen. Dies bedeutet für die universitäre Lehre: Was früher Examenswissen war, ist heute Grundstudiumswissen. Wo früher die sprachgeschichtliche Ausbildung geendet hat, bildet sie heute Ausgangspunkt für weiterführende, spannende Fragen. Soviel zu dem häufig zu vernehmenden Eindruck, dass die sprachhistorische Ausbildung früher besser und fundierter war. Aus eigener Erfahrung füge ich hinzu: Sie war außerdem langweilig, Vieles hat sich wiederholt, der Blick auf das Ganze hat gefehlt. Die neue Erfahrung lehrt: Die Studierenden akzeptieren trotz heutzutage deutlich größerer Arbeitsbelastung nicht nur ein höheres Ni- 
veau, sie schätzen es auch, was sich an ihrer generell hohen Leistungsbereitschaft, an anspruchsvollen Diskussionen und guten Haus- und Abschlussarbeiten zeigt. Auch wird immer wieder von Lehramtsstudierenden die Absicht geäußert, vermehrt sprachhistorische Einheiten in den Unterricht einbringen $\mathrm{zu}$ wollen: Sprachgeschichte wird als wichtig begriffen. Themen, die sich besonders für Lehramtsstudierende eignen und daher in den universitären Unterrichtskanon gehören, sind die Onomastik (vorrangig, doch keineswegs zwingend, die Anthroponomastik), die sog. Zweifelsfall-Linguistik, die nach dem Hintergrund aktueller Schwankungsfälle fragt und dabei nicht selten auf (schon im Frühnhd. fußenden) Sprachwandel stößt, die Phraseologie, aber auch Veranstaltungen zur Grammatikalisierung, zum Sprachwandel prinzipiell, zur historischen Graphematik, Phonologie, Morphologie, Syntax, Semantik, Pragmatik. Ein Motor sprachlichen Wandels besteht in der Tatsache, dass jede sprachliche Ebene sich nach spezifischen Optimierungsprinzipien wandelt und dabei andere Ebenen beeinträchtigen kann. Einen solchen Antagonismus werden wir mit dem Beispiel des phonologischen und des morphologischen Wandels kennenlernen (vgl. Abschnitt 2 und 3). Die Historische Linguistik braucht sich also nicht zu verstecken oder zu rechtfertigen, wenn sie lebensnah vermittelt wird.

Eine weitere Bereicherung und - buchstäblich - Horizonterweiterung erfährt die Historische Sprachwissenschaft durch die Wahrnehmung der Sprachtypologie, so wie umgekehrt die Sprachtypologie durchaus und zunehmend an sprachhistorischen Erkenntnissen interessiert ist, auch wenn sie (die Typologie) - oft zwangsläufig - strikt synchron-vergleichend verfährt und dabei meist - für Philologen oft schwer erträglich - sehr grobrastrig vorgeht. Dennoch tun sich interessante Verbindungen auf. Croft (2003) spricht im Zusammenhang diachroner Forschung von einer „dynamicization of typology“ (232ff., vgl. auch Ineichen 1991: 123ff.). Rekonstruierte Systeme (z. B. Lautsysteme) können angesichts der Kenntnis sprachtypologischer Verbreitungen als mehr oder weniger plausibel bewertet werden. Umgekehrt kann die Historische Sprachwissenschaft, indem sie genau die Dynamik sprachlicher Veränderung erforscht, z. B. zeigen, welche Merkmale bei einem typologischen Wandel früher/später ab- oder aufgebaut werden, wie die Implikationen zwischen den Merkmalen beschaffen sind, welche typologischen Merkmale eher hart oder weich sind, kurz: in welcher Sukzession sich ein Sprachtyp auf- oder abbaut.

Ich möchte, um konkret zu werden, drei Beispiele für den produktiven Dialog zwischen Historischer Sprachwissenschaft und Sprachtypologie liefern: 1. Den phonologisch-typologischen Wandel des Deutschen von einer Silben- zu einer Wortsprache, 2. die frühnhd. ,Justierung' der Abfolge grammatischer Kategorien am Verb gemäß der universellen Rele- 
vanzskala, und 3. die Entwicklung unseres Höflichkeitssystems am Beispiel der Anredepronomen. Weder liefere ich Neues noch kann ich ins Detail gehen. Es geht hier nur darum, für die gegenseitige Wahrnehmung und Zusammenarbeit linguistischer Disziplinen zu werben.

\section{Präteritum(s?)schwund, Subjekt(s?)pronomen und}

\section{Merkmal(s?)analyse: Der phonologisch-typologische Wandel des}

Deutschen von einer Silben- zu einer Wortsprache - am

Beispiel heute schwankender Fugenelemente

Heutige Zweifels- oder Schwankungsfälle, die nach der Definition von Klein $(2003,2009)$ von erwachsenen Muttersprachlern bemerkt und reflektiert werden, können in gewisser Hinsicht mit Beben verglichen werden: Es finden (die Menschen stark verunsichernde und beunruhigende) Verwerfungen statt, die auf Verschiebungen tiefliegender tektonischer Platten zurückgehen. Was sich mit welchem Tempo wohin verschiebt und weshalb, ist nicht sichtbar.

Einer der größten und bei Sprachberatungsstellen am häufigsten nachgefragten Zweifelsfälle bildet die $s$-vs. Null- oder Nichtverfugung von Komposita wie in der Überschrift genannt, doch ließen sich diese Beispiele mühelos vervielfachen: Sie gehen in die Hunderte. Einige weitere Beispiele: Interessen(s?)bekundung, Respekt(s?)person, Denkmal(s?)pflege, Lehramt(s?)kandidat, Seminar(s?)arbeit, Antrag(s?)formular, Erbschaft(s?)steuer. Allein in der linguistischen Fachterminologie gibt es mehr als ein Dutzend solcher Fälle.

Man hat lange nach den Gründen für diese Unsicherheit der $s$ Verfugung gesucht (die bei diesem Prozess auf Kosten der Nullfuge zunimmt) und kam dabei auf so abwegige Erklärungen, dass es einfach besser klinge, wenn da ein -s- stünde (dies wurde nicht nur von Bastian Sick vertreten) bzw. dass das Wort leichter mit $-s$ - aussprechbar sei (Busch/ Stenschke 2007: 87). Letzteres kann leicht zurückgewiesen werden: Jedes -s- im Wortauslaut führt zu einem phonologischen Komplexitätszuwachs - und genau hierin besteht die eigentliche Begründung für das -s-, allerdings von ganz anderer Seite her argumentierend (s. u.).

Zunächst seien die beiden bekanntesten Begründungen für die Setzung der $s$-Fuge referiert, die morphologischer Natur sind.

Erstens: Es gibt sog. schließende Suffixe, d. h. solche, die keine weitere Derivation erlauben. Dies gilt für -ung, -sal, -in, -ion, -ling, bedingt auch für -schaft und -heit (Aronoff/Fuhrhop 2002), vgl. Liebling, aber *lieblinglich, *Lieblingin etc. Solche Suffixe werden durch die $s$-Fuge für die Kompositi- 
on geöffnet: Liebling-s-essen. Allerdings erfasst dieses Prinzip der Öffnung morphologisch schließender Suffixe für die Komposition nur einen kleinen (den obigen) Ausschnitt der Fugenvorkommen.

Zweitens: Je morphologisch komplexer das Erstglied sei, desto eher werde dieses verfugt (Henzen 1965, Ortner et al. 1991, Fuhrhop 1996, 1998). Damit diene die $s$-Fuge der Segmentierung morphologisch besonders komplexer Wörter. Tatsächlich deuten zahlreiche Kompositapaare vom Typ Hofmaner, aber Friedhof-s-mauer, Kaufpreis, aber Verkauf-s-preis, Fabrtzeit, aber Abfahrt-s-zeit darauf hin. Allerdings hat Kürschner (2003) bei einer Korpusanalyse festgestellt, dass morphologisch besonders komplexe Erstglieder, nämlich solche, die selbst ein Kompositum bilden, aus dieser Komplexitätsregel ausscheren (Tab. 1).

Tab. 1: Abhängigkeit von Null- und -s-Fuge von der morphologischen Komplexität des Erstglieds (nach Kürschner 2003)

\begin{tabular}{|c|c|c|c|}
\hline \multirow{2}{*}{ Fugenelement } & $\begin{array}{c}\text { alle } \\
\text { Komposita }\end{array}$ & \multicolumn{2}{|c|}{$\begin{array}{c}\text { Komposita mit } \\
\text { polymorphemischen Erstgliedern }\end{array}$} \\
\cline { 3 - 4 } & & $\begin{array}{c}\text { Erstglied ist } \\
\text { Kompositum }\end{array}$ & $\begin{array}{c}\text { Erstglied ist derivationell } \\
\text { komplex }\end{array}$ \\
\hline Null $\varnothing$ & $\mathbf{5 8 \%}$ & $\mathbf{6 6} \%$ & $29 \%$ \\
\hline$-s-$ & $25 \%$ & $27 \%$ & $\mathbf{6 7 , 5 \%}$ \\
\hline
\end{tabular}

Werden im Schnitt alle Komposita zu 58\% nullverfugt und zu 25\% sverfugt (die $s$-Fuge ist das häufigste von insgesamt sechs Fugenelementen), so ändert sich dies kaum, wenn das Erstglied seinerseits ein Kompositum bildet. Beispiele: Bilderbuch-Ø-wetter und Rückruf-Ø-aktion. Nur dann, wenn die morphologische Komplexität auf Derivation basiert, schnellt die $s$ Verfugungsrate von $25 \%$ auf 67,5\% hoch (Beruf-s-wunsch, Absicht-serklärung). Also kann es sich nur um eine spezifische Form morphologischer Komplexität handeln, die die $s$-Fugensetzung begünstigt.

Eine weitere morphologische Begründung, nämlich die, dass es sich bei der $s$-Fuge um Genitivflexive handle, muss angesichts zu vieler Gegenbeispiele verworfen werden: Weder handelt es sich bei einem Freundeskreis um den *'Kreis eines Freundes' noch bei dem Lieblingsessen um das ${ }^{*}$ 'Essen des/eines Lieblings'. Schließlich treten die meisten $s$-Fugen unpa- 
radigmisch auf, allen voran alle die (und das sind die meisten), die auf feminine Erstglieder folgen (Abfahrt-s-zeit): Hier wäre es unsinnig, von Flexiven zu sprechen.

Wie in Nübling/Szczepaniak (2008, 2009) gezeigt, ist die Fugensetzung prosodisch-phonologisch gesteuert: Anhand einer großen Korpusuntersuchung kamen wir zu dem Ergebnis, dass in dem Maße, in dem das Erstglied von dem für das Deutsche geltenden phonologischen Wortideal des einfüßigen Trochäus mit Reduktionssilbe (Typ Mutt[e], Kann[ə]) abweicht, das Erstglied mit -s- verfugt wird. Das heißt: Je phonologisch ,schlechter das Erstglied und je schwieriger damit seine Wortgrenzen perzipierbar sind, desto eher wird sein rechter Wortrand markiert (verfugt), vgl. Abb. 1. Die s-Fuge ist damit ein Signal schlechter phonologischer Wortqualität. Umgekehrt werden Trochäen mit Reduktionssilbe am seltensten $s$-verfugt. Dies geschieht nur dann, wenn es sich um deverbale Nomina handelt, d. h. um solche, die mit Infinitiven homophon und daher mit diesen verwechselbar wären: Wissen-s-bestand, Glücken-s-bedingung, Verbalten-s-weise.

phonologische

Wortqualität:
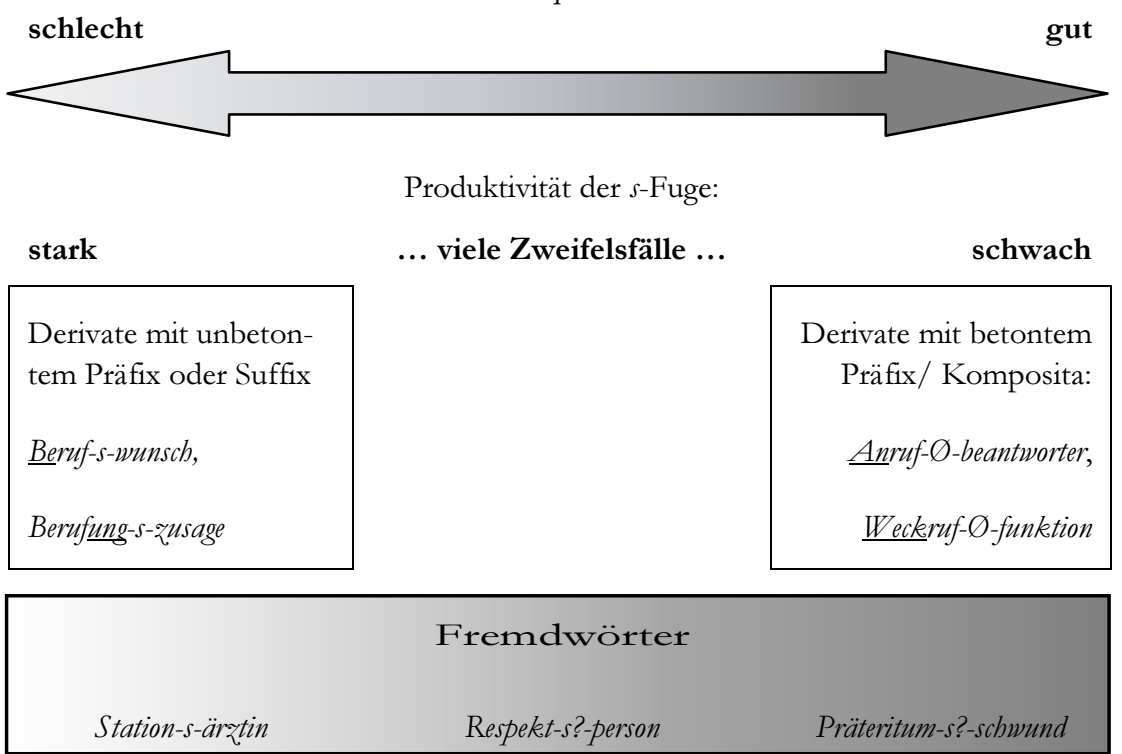

Abb. 1: Die Abhängigkeit der s-Fugensetzung von der phonologischen Wortqualität des Erstglieds 
Diese auf die Wortqualität bezogene phonologische Regel erklärt z. B., weshalb besonders viele $s$-verfugte Erstglieder Fremdwörter sind. Regelmäßig werden finalbetonte auf -ität und -ion (aber auch viele andere) verfugt, denn diese divergieren durch Mehrfüßigkeit, Vollvokale in unbetonten Silben und Nicht-Initialakzente am stärksten vom Erbwortschatz (zu Näherem vgl. Nübling/Szczepaniak 2009). Doch auch die bisher morphologisch (partiell) begründeten Sachverhalte werden phonologisch besser erfasst und erklärt: Die morphologisch schließenden Suffixe sind nichts anderes als nebenbetonte Suffixe mit Vollvokal (im Fall von -ung sogar unbetont mit Vollvokal), d. h. Hybride, deren Zugehörigkeit zum Erstglied durch die $s$-Setzung unterstrichen wird. Schließlich erzeugen auch die derivationell komplexen Erstglieder (im Unterschied zu den kompositionell komplexen) phonologische Komplexität, indem gerade die unbetonten Präfixe für nichttrochäische und (hinten) schwa-lose Strukturen sorgen, daher Rufmord, aber Berúf-s-wunsch, Kaufpreis, aber Verkáuf-s-preis. Dieser Typus wird zu über $80 \%$ verfugt und generiert damit eher wenige Zweifelsfälle. Nur zu 36\% verfugt und eine Quelle von Zweifelsfällen sind die Erstglieder mit betontem Präfix bzw. grundsätzlich solche Bildungen, die zwar trochäisch strukturiert sind, aber hinten keine Reduktionsvokale enthalten: Merkmal(s?)analyse, Denkmal(s?)pflege, Antrag(s?)formular. Auch auffällig viele Fremdwort-Erstglieder befinden sich unter den Zweifelsfällen.

Was haben diese Befunde mit der Typologie zu tun? Sehr viel, denn, um im Bild zu bleiben, bildet sie die tektonischen Platten darunter. Wie der Beitrag von Renata Szczepaniak in diesem Band zeigt, hat das Deutsche einen tiefgreifenden phonologischen Wandel von einer ahd. Silbenzu einer nhd. Wortsprache durchlaufen - und fährt auch heute noch fort, die Wortsprachlichkeit auszubauen (vgl. ausführlich Szczepaniak 2007 und einführend Szczepaniak 2008: 11-41). Während die phonologischen Prozesse des Althochdeutschen (und Westgermanischen) allesamt auf eine Optimierung der universell gültigen Idealsilbe CV hinauslaufen (westgerm. Konsonantengemination, ahd. $i$-Umlaute, ahd. Vokalharmonien epenthetischer Vokale, Vorkommen von Vokal- und Konsonantenepenthesen, Assimilationen, Notkersches Anlautgesetz) oder zumindest silbenbezogen verlaufen (die gesamte 2. Lautverschiebung), vollzieht sich zum Mhd. hin in vielerlei Hinsicht eine Verschlechterung der Silbenstruktur (Endsilbenabschwächung, Aufspaltung/Asymmetrisierung des Haupt- und Nebentonvokalismus, Syn- und Apokopen, Entstehung komplexer Konsonantengruppen) und gleichzeitig, besonders zum (Früh-)Nhd. hin, ein Aufbau an Wortsprachlichkeit, d. h. die phonologischen Prozesse profilieren zunehmend die Informationseinheit Wort (oder Morphem) und/oder wählen es zu ihrer Bezugsdomäne. Dies beginnt zunächst mit der Regulierung 
und Stabilisierung der Wortgröße zum Trochäus ab mhd. Zeit sowie mit dem Abbau der Geminaten zur gleichen Zeit. Dazu gehört auch die Phonologisierung der Umlautprodukte, die Entstehung ambisilbischer Konsonanten, die frühnhd. Dehnung in offener Tonsilbe - bis hin zu Konsonantenepenthesen, die nunmehr das genaue Gegenteil einer CVOptimierung bewirken, nämlich die rechte Wortrandverstärkung: mhd. ieman > nhd. jemand, saf $>$ Saft, obez > obst, mâne > Mond etc. Die Wortränder werden außerdem (vorne) durch die Aspiration anlautender prävokalischer Plosive, durch die Entstehung des Glottisverschlusses und (hinten) durch die Auslautneutralisierung, durch die heute massenhaft entstehenden silbischen Nasale und Liquide sowie die Verletzung der Sonoritätshierarchie durch extrasilbische Konsonanten in Aus-, aber auch Anlautclustern profiliert: [jt]adt, O[pst].

Genau hierein fügen sich die Fugenelemente, und zwar nicht nur, indem sie die geringe wortphonologische Qualität des Erstglieds signalisieren, sondern indem sie sozusagen aktiv zusätzlich zur Verschlechterung eben dieses Wortauslauts beitragen durch die Verstärkung des rechten Wortrands: Wie auch schon Wegener (2006) anhand monosyllabischer Erstglieder festgestellt hat, tritt das frikative Fugen-s besonders dann gerne an das Erstglied an, wenn es Extrasyllabizität erzeugt, d. h. wenn es konsonantisch stärkeren Lauten (also Plosiven) folgt: Ort-s-zeit, Wirt-s-baus, auch Geburt-s-tag, Abfahrt-s-zeit, Ankunft-s-zeit, Meisterschaft-s-favorit, Mehrheits-meinung, Kind-s-kopf, Verbund-s-lösung, Geduld-s-faden. Überall hier erweitert das Fugen- $s$ nicht einfach nur den Konsonantencluster, sondern es verschlechtert ihn durch die Verletzung des kontinuierlichen Zuwachses an konsonantischer Stärke. Nicht zufällig, so muss man schlussfolgern, hat von den sechs Fugenelementen ausgerechnet $-s$ - das Rennen gemacht. Eine Option wäre gewesen, mit dem einstigen Allomorph -es- zu alternieren und damit Trochäen zu erzeugen (was $-n$ - und -en- leisten). Genau dieser Weg wurde nicht eingeschlagen, da typologisch schon eine andere Richtung eingeschlagen war: Der heutige wortsprachliche Ausbau besteht darin, den rechten Wortrand auszubauen, komplexer zu machen. Nur wenn man diese typologische Drift des Deutschen kennt, versteht man das heutige Fugenverhalten mit all seinen Schwankungsfällen, die von nichts anderem als gegenwärtig sich vollziehendem Sprachwandel zeugen.

Diese prosodisch-phonologische Typologie von Silben- versus Akzent- bzw. Wortsprachen wurde schon länger beschrieben und von Auer (2001) präzisiert und weiterentwickelt. Mit der Arbeit von Szczepaniak (2007) wurde sie erstmals sprachgeschichtlich nutz- und fruchtbar gemacht bzw., in den Worten von Croft, dynamisiert. Auf diese Weise lässt sich ermitteln, wie im Einzelnen der (ahd.) silben- bzw. (fnhd.) wortsprachliche Ausbau (aber auch der silbensprachliche Abbau im Mhd.) 
abläuft, welche Merkmale es genau sind, die früher auftreten und welche erst später. Schließlich zeigt sich, dass der wortsprachliche Ausbau nur anfänglich in der Vernachlässigung und Verschlechterung der Silbe besteht und später zu ganz anderen Maßnahmen greift. Es existiert in der deutschen Sprachgeschichte übrigens kein einziger phonologischer Wandel, der sich nicht in diese typologische Drift einfügte. So erfahren alle über Generationen hinweg auswendig gelernten und kaum verstandenen phonologischen Prozesse eine übergreifende Einordnung und Erklärung und dies gilt auch für manchen aktuellen Zweifelsfall.

\section{Die frühnhd. Justierung ' der Abfolge grammatischer Kategorien am Verb gemäß der universellen Relevanzskala}

Die deutsche Sprachgeschichte ist, wie der vorige Abschnitt gezeigt hat, viele Jahrhunderte lang von phonologischem Wandel dominiert gewesen. Phonologischer Wandel folgt anderen Gesetzen als morphologischer Wandel. Es nimmt daher wenig wunder, dass diese phonologischen Prozesse destruktive Auswirkungen auf die Morphologie hatten. Erst nach mhd. Zeit beginnt die Morphologie, sich zu reorganisieren und die Folgen des Lautwandels zu ,reparieren'. Nach welchen Prinzipien dies erfolgt, erweist ein Blick auf die verbalflexionsmorphologische Typologie, wie sie von Bybee (1985) anhand des synchronen Vergleichs von 50 verschiedenen (weder areal noch ,genetisch' zusammengehörigen) Sprachen erarbeitet wurde. Ihre daraus abgeleitete Relevanzskala verbaler Kategorien erfährt eine zuvor ungeahnte Bestätigung durch die Diachronie des Deutschen: Streng relevanzgesteuert treten bei diesem im Frühnhd. stattfindenden morphologischen Umbau kategorielle Stärkungen relevanter (Tempus, Modus) sowie Schwächungen weniger relevanter Kategorien (Numerus, Person) ein. Dabei erweist sich die sog. Tempusprofilierung bis heute als das flexionsmorphologische Leitmotiv des deutschen Verbs. Bybee selbst hat in ihrem Beitrag von 1994 „Morphological universals and change" ihre synchron-typologisch gewonnenen Befunde auf die Diachronie bezogen, wenngleich nicht auf die deutsche und wenig detailliert. Dies soll im Folgenden getan werden (vgl. ausführlich Nübling/Dammel 2004).

Bei der typologischen Untersuchung erwiesen sich als die weltweit häufigsten Verbalkategorien Person, Numerus, Modus, Tempus, Aspekt, Diathese und Valenz. Das Deutsche realisiert nur die ersten vier flexivisch, weswegen wir uns nur auf diese beschränken. Die formale Abfolge dieser vier Kategorien am Verb folgt zuvörderst dem sog. Relevanaprinæip, womit sog. diagrammatischer Ikonismus entsteht: 
A meaning element is relevant to another meaning element if the semantic content of the first directly affects or modifies the semantic content of the second. If two meaning elements are, by their content, highly relevant to one another, then it is predicted that they may have lexical or inflectional expression, but if they are irrelevant to one another, then their combination will be restricted to syntactic expression. (Bybee 1985: 13; Hervorhebungen im Original)

Numerus und Person sind weniger relevant, da sie nur die Aktanten der Handlung bezeichnen, die die Aktion zwar ausführen, sie jedoch nicht in ihrer Beschaffenheit modifizieren. Außerdem wird der Aktant üblicherweise durch ein nominales oder pronominales Subjekt ausgedrückt, weshalb Bybee hier von sog. ,agreement categories“ (Bybee 1985: 28) spricht. Demgegenüber modifizieren andere Kategorien durchaus die Verbalhandlung selbst: So bezieht sich Aspekt (der heute nicht mehr flexivisch realisiert wird) auf ihren internen Verlauf, indem er verschiedene Phasen fokussiert (inchoativ/ingressiv, durativ, resultativ/perfektiv etc.). Anders Tempus, dem zwar auch ein hoher Relevanzgrad zukommt, das aber die durch das Verb bezeichnete Handlung nur extern temporal situiert, sie als solche jedoch intakt lässt: Die Handlung wird nur in verschiedene Zeitstufen transponiert. Modus bezeichnet im weitesten Sinn die Haltung des Sprechers zum Sachverhalt, d. h. zur gesamten Proposition; sie verändert nicht die Semantik des Verbs, sondern macht Aussagen über den Faktizitätsgrad der Proposition, wie ihn der Sprecher einschätzt, oder zur Quelle, aus der sein das Wissen bezieht. Im Imperativ fordert der Sprecher zum Vollzug der Handlung auf.

Allerdings werden diese (und die hier nicht genannten) unterschiedlich relevanten Informationen in den Sprachen der Welt keineswegs alle flexivisch ausgedrückt. Hier ist das sog. Allgemeingültigkeitsprin₹ip (generality) zu berücksichtigen, das einen möglichst geringen semantischen Gehalt der Kategorie vorsieht, um sie damit umso kompatibler für die Verbbedeutung zu machen:

However, generality distinguishes inflectional from all the rest. Inflectional categories are more general - have a wider range of applicability with predictable meaning - than lexical, derivational, or periphrastic categories. Thus generality is a necessary defining feature of inflection (Bybee et al. 1994: 22).

Damit ergibt sich für das Deutsche eine Relevanzskala wie in Abb. 2 (S. 73). Wenn der Relevanz- oder der Allgemeingültigkeitsgrad zu hoch ist, wird - je nachdem - die betreffende Kategorie über andere formale Verfahren kodiert (lexikalisch, derivationell, syntaktisch).

Der dritte Faktor, der dieses Funktions-Form-Verhältnis maßgeblich steuert, ist die Tokenfrequens, die sich a) in die lexikalische und b) in die kategorielle oder grammatische Frequenz aufspaltet: a) geben als Verblexem 
kommt bedeutend häufiger vor als beben; b) grammatische Kategorien(kombinationen) werden unterschiedlich häufig aktiviert: So ist die 3.Sg.Präs. viel häufiger als die 2.Pl.Prät. (zu diesen Frequenzen vgl. Tomczyk-Popińska 1987). Hohe Tokenfrequenz wirkt auf den Ausdruck grammatischer Kategorien prinzipiell fusionierend (komprimierend), d. h. sie sorgt, unabhängig vom Relevanzgrad, für Linksverschiebungen auf der Skala in Abb. 2.

\begin{tabular}{|lrr|}
\hline Tempus & Numerus & Modus \\
& & \\
+ Relevanz & Person \\
- Allgemeingültigkeit & Allgemeingültigkeit \\
fusionierender Ausdruck & \\
mehr Allomorphie & \\
\hline
\end{tabular}

Abb. 2: Die Flexionskategorien des Verbs im Deutschen nach Relevanz- bzw. Allgemeingültigkeitsgrad und ihr Bezug zu Fusion und Allomorphie

Abb. 3 (S. 74) liefert ein Beispiel dafür und zeigt, dass das Tempusmorphem in einem selteneren Verb (wie beben) additiv markiert wird (wenngleich das Dentalsuffix der hohen Relevanz von Tempus wegen in direkte Nachbarschaft zum Stamm tritt), während frequente Verben (wie geben) die Tempusinformation (trotz gleichen Relevanzgrads) direkt in den lexikalischen Stamm integrieren (Ablaut), hier also echte Fusion (Wurzelflexion) zulassen.

Die wenig relevante Person/Numerus-Endung steht dagegen bei beiden Verbformen in der Peripherie. Insgesamt ist gabst deutlich kürzer als bebtest, was dem Prinzip formaler Kürze bei Hochfrequenz entspricht. Der nach den langen Phasen phonologischen Wandels einsetzende morphologische Wandel bestätigt auf fast vorbildhafte Weise die Gültigkeit dieser drei Prinzipien: Im Frühnhd. werden die minderrelevanten Kategorien (Person, Numerus) geschwächt und die relevanten (Modus, Tempus) gestärkt, im Einzelnen abhängig von den konkreten Frequenzen. Abb. 4 (S. 74) zeigt das gesamte Bild, das hier nur auszugsweise behandelt werden kann (vgl. eingehend Nübling/Dammel 2004). 
schwache Verben: beben
(geringe lexikal. Frequenz)

Relevanz

'beben' 'Prät.' '2.Sg.'

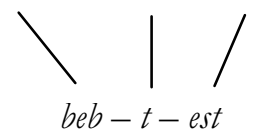

starke Verben: geben

(hohe lexikal. Frequenz)

Relevanz

'geben' 'Prät.' '2.Sg.'

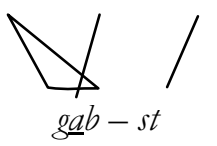

Abb. 3: Frequenzbedingte Markierungsunterschiede beim Präteritum von beben und geben

Stärkung, Profilierung

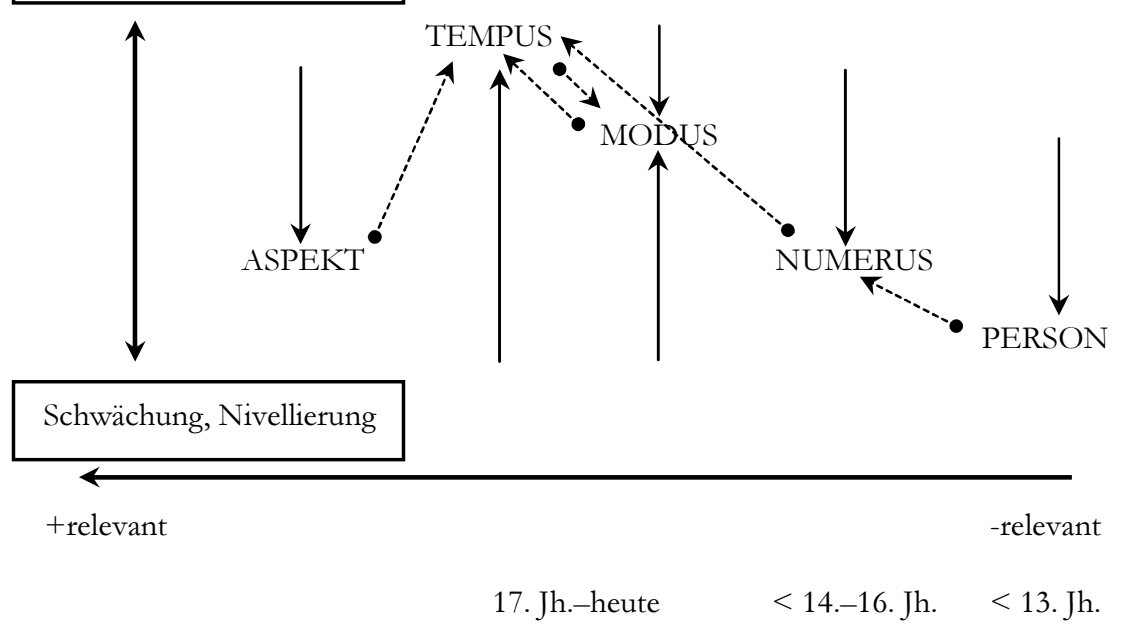

Abb. 4: Übersicht über relevanzgesteuerte Stärkungen und Schwächungen verbaler Kategorien im Deutschen

Im Folgenden wird aus diesem Komplex nur 1.) die Schwächung von Person sowie 2.) die Stärkung von Tempus herausgegriffen.

1.) Schwächung/Nivellierung von Person, hier nur am Beispiel der 2.Sg.: Der blind wirkende phonologische Umlaut hat zu morphologischen 
Verzerrungen geführt. So hat die ahd. Endung $-i$ der 2.Sg.Prät. starker Verben den Stammvokal umgelautet: ahd. bundi $>b[y] n d i>$ mhd. bünde 'bandst'. Aus phonologischer Perspektive handelt es sich um eine regressive Fernassimilation, die die Silbennuklei artikulatorisch einander annähert, während aus morphologischer Perspektive diese Person/NumerusInformation in die Wurzel gelangt - und dort auch verbleibt, nachdem der Umlaut im Mhd. phonologisiert war. Damit hat eine wenig relevante Kategorie einen formal-fusionierenden Ausdruck erlangt, wie dies nur hochrelevanten Kategorien gebührt. Darüber hinaus hatte die 2.Sg.Prät. einen vom Sg.-Paradigma abweichenden Ablaut. Zum Flexiv der 2.Sg. gab es noch zwei weitere Allomorphe, $-t$ im Präsens von Präteritopräsentia $(d u$ wilt $t$ und $-s t$ sonst. Schon im frühen Frühnhd. wird der Umlaut aus der 2.Sg.Prät. beseitigt, dabei auch noch die Ablautstufe des Präteritum Singular übernommen und außerdem das häufigste Allomorph -st. $d u$ bünde $>d u$ band(e)st. Damit war die Personenkategorie aus dem Stamm beseitigt. Im späteren Frühnhd. wird die $t$-Endung der Präteritopräsentia - ganz der lexikalischen Frequenz dieser Verben folgend - durch nun uniformes -st ersetzt: $d u$ wilt $>d u$ willst. Dies zeigt Tab. 2 (S. 76). Auch der Grad an Allomorphie ist relevanzabhängig: Bei hoher Kategorienrelevanz leisten sich die Sprachen viel, bei geringer wenig Allomorphie (vgl. Abb. 2). Auch diesbezüglich hat eine, Justierung' des Personenausdrucks stattgefunden.

2.) Stärkung/Profilierung von Tempus: Durch die Entwicklung von mhd. bünde $>$ fnhd. band(e)st wird - zunächst - Numerus gestärkt insofern, als nun Ablautstufe 2 für den Singular zuständig ist (band/bandest/band) und Ablautstufe 3 ausschließlich den Plural (bunden/bundet/bunden). Die Numerusstärkung (vgl. den gestrichelten Pfeil von Person zu Numerus in Abb. 4) bestand jedoch nur vorübergehend, wie Tab. 3 (S. 76) zusammenfasst: Der präteritale Numerusausgleich, die wohl größte morphologische Umwälzung im Frühnhd., vernichtet bald wieder diese wurzelinterne (ablautende) Numerusopposition, und zwar zugunsten von Tempus. In dem Moment, in dem der Ablaut sich aus dem Numerus (im Prät.) zurückzieht, ist es einzig und allein noch die relevante Tempuskategorie, die von diesem salienten, wurzelinternen Ausdrucksverfahren Gebrauch macht: Der Rückzug von Numerus aus der Verbwurzel dient (,passiv') der Stärkung von Tempus (vgl. den gestrichelten Pfeil von Numerus zu Tempus in Abb. 4). Weitere Faktoren, die den präteritalen Numerusausgleich begünstigt haben, werden in Nübling (1998) diskutiert; hierzu gehört u. a. der sich im Frühnhd. anbahnende Präteritumschwund, der eine rückläufige Kategorienfrequenz und damit den Abbau morphologischer Differenzierungen bewirkt. 
Tab. 2: Schwächung von Person durch Reduktion von Allomorphie: die 2. Person Singular

\begin{tabular}{|c|c|c|c|}
\hline & \multicolumn{3}{|c|}{ '2.Sg.' } \\
\hline $\begin{array}{c}\text { Flexionsklasse/ } \\
\text { Kategorien- } \\
\text { kombination }\end{array}$ & $\begin{array}{l}\text { st. Vb./ } \\
\text { Ind.Prät. }\end{array}$ & $\begin{array}{c}\text { Präteritopräs./ } \\
\text { Ind.Präs. }\end{array}$ & sonst \\
\hline $\begin{array}{l}\text { Allomorphe im } \\
\text { Mhd. }\end{array}$ & $\begin{array}{c}\{\mathrm{Pl}-\mathrm{AL}, \mathrm{UL}+-\mathrm{e}\} \\
(d u) \text { bünd } \underline{e}\end{array}$ & $\begin{array}{c}\{-\mathrm{t}\} \\
(d u) \text { wilt }\end{array}$ & $\begin{array}{c}\{-s t\} \\
\text { (du) gib } \underline{s t}\end{array}$ \\
\hline $\begin{array}{l}\text { uniformes Morphem } \\
\qquad \text { im Nhd. }\end{array}$ & \multicolumn{3}{|c|}{$\begin{array}{c}\{-s t\} \\
d u \text { bandst, willst, gibst }\end{array}$} \\
\hline
\end{tabular}

Tab. 3: Von der Personennivellierung zur Numerusprofilierung und von der Numerusnivellierung zur Tempusprofilierung

\begin{tabular}{|c|c|c|c|c|}
\hline \multicolumn{2}{|c|}{ Prät.Ind. } & Mhd. & Frühnhd. & Nhd. \\
\hline \multirow{3}{*}{$\mathrm{Sg}$. } & 1. & bant & band & band \\
\hline & 2. & bünd-e & band-est & band-est \\
\hline & 3. & bant & band & band \\
\hline \multirow{3}{*}{ Pl. } & 1. & bund-en & bund-en & band-en \\
\hline & 2. & bund-et & bund-et & band-et \\
\hline & 3. & bund-en & bund-en & band-en \\
\hline & & $\begin{array}{c}\text { kein klares } \\
\text { System } \\
\text { (Plural relativ } \\
\text { homogen) }\end{array}$ & $\begin{array}{c}\text { Personennivellierung }> \\
\text { Numerusprofilierung } \\
\text { (Numerusablaut): } \\
\text { band vs. bunden }\end{array}$ & $\begin{array}{c}\text { Numerusnivellierung } \\
\text { (prät. Numerusausgleich) } \\
>\text { Tempusprofilierung: } \\
\text { binden vs. bạnd vs. } \\
\text { gebunnden }\end{array}$ \\
\hline
\end{tabular}

Neben dieser ,passiven' Stärkung von Tempus durch Rückzug von Numerus aus dem Ablautverfahren erfährt die Tempuskategorie weitere, ,aktive Profilierungen, z. B. durch die Fragmentierung des Ablautsystems, die umgekehrt als Tempusallomorphiezuwachs zu bewerten ist und von der Stärke der Tempuskategorie zeugt. Hinzu kommt die hohe lexikalische Frequenz, die auch heute noch den ca. 150 verbleibenden starken Verben zukommt, nachdem minderfrequente in die schwache Klasse abgewandert sind (Augst 1975). Wie es im Einzelnen zur Entstehung von heute über 
40 Ablautalternanzen aus dem mhd. Siebenreihensystem kam, hat sowohl a) morphologische als auch b) phonologische Gründe. Zu jedem nur ein Beispiel: a) Dass werden - wurde - geworden heute ein (extrem tokenfrequenter) Einzelgänger ist, liegt (unter anderem) an der anderen Ausgleichsrichtung, die dieses Verb beim präteritalen Numerusausgleich gewählt hat: Während üblicherweise die 2. Stufe (die einstige Prät.Sg.-Stufe) generalisiert wurde (vgl. werfen - warf - geworfen), hat dieses Verb zur 3. Stufe (dem Prät.Pl.) gegriffen. b) Auch kommen - kam - gekommen ist heute ein (extrem tokenfrequenter) Einzelgänger, der auf die singuläre Assimilation von ahd. queman > mhd. komen zurückgeht (progressive Labialisierung von $e>0$ durch $[\mathrm{w}])$. Bei quellen ist dies beispielsweise nicht eingetreten (zu solchen Irregularisierungsstrategien vgl. Nübling 2000).

Im Gegensatz zu Bybee (1994), auch Bybee et al. (1994), die in ihrem diachronen Ansatz die Genese von relevanzgesteuerter Morphologie über Grammatikalisierungen verfolgt, also neu entstehende, junge Morphologie zum Thema hat, zeigt das Beispiel der deutschen Sprachgeschichte, dass sich auch längst vorhandene, d. h. alte, doch ,verzerrte' Morphologie reorganisieren kann - und dies exakt dem Relevanzprinzip folgend: Es gibt $\mathrm{m}$. W. keinen morphologischen Wandel im (Früh-)Nhd., der dieses Prinzip konterkariert. Dies gilt auch für die Nominalmorphologie. Überdies hat sich auch gezeigt, wie Schwächungen der einen Kategorie Stärkungen einer anderen bewirken können, d. h. es besteht komplexe Interaktivität zwischen den Kategorien und ihren Markern. Solche nur über die diachrone Forschung zu gewinnenden Beobachtungen und Einsichten bereichern die typologische Forschung.

\section{Die Entwicklung des deutschen Höflichkeitssystems am Beispiel der Anredepronomen}

Ein Musterbeispiel für die Synthese allgemein-sprachtypologischer Erkenntnisse mit konkret-sprachgeschichtlichem Wandel liefert Simon (2003a) mit der Untersuchung Für eine grammatische Kategorie ,Respekt' im Deutschen. Synchronie, Diachronie und Typologie der deutschen Anredepronomina (vgl. auch Simon 1997 und 2003b). Wieder können wir nur einen Aspekt herausgreifen, der speziell die Entwicklung des deutschen Anredepronominalsystems betrifft und es erstmals überzeugend erklärt. Der Verlauf ist in Tab. 4 (S. 78) zusammengefasst: Ein im Germanischen vermutlich einstufiges Adressatensystem entwickelt sich nach und nach zu einem mehrstufigen, bis hin zu einem fünfstufigen im frühen Nhd. Heute ist mit der Opposition $d u /$ Sie ein zweistufiges System erreicht. Dass immer mehr Höflichkeitsstufen hinzukamen, wird erstens mit dem inflationären Ge- 
brauch bisheriger Höflichkeitsformen begründet, zweitens mit gesellschaftlichem Wandel (Ständegesellschaft), der sich direkt im Anredesystem abbildet, und drittens mit Sprachkontakt v. a. zum Lateinischen (vos) und Französischen (vous), der besonders für das Ihrzen ab dem Ahd. verantwortlich gemacht wird (vgl. z. B. Besch 1996, 2003).

Tab. 4: Diachronie der pronominalen Anrede einer Einzelperson (aus Dammel 2008 nach Simon 2003a)

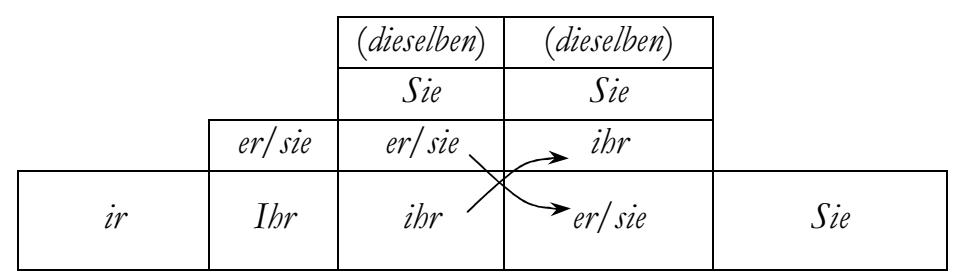

\begin{tabular}{|c|c|c|c|c|c|}
\hline$d u$ & $d u$ & $D u$ & $d u$ & $d u$ & $d u$ \\
\hline \hline Germ. & $\begin{array}{c}\text { Ahd.- } \\
\text { Fnhd. }\end{array}$ & 17. Jh. & 18. Jh. & $\begin{array}{c}\text { frühes 19. } \\
\text { Jh. }\end{array}$ & Nhd. Std. \\
\hline Stufe 1 & Stufe 2 & Stufe 3 & Stufe 4 & Stufe 5 & Stufe 6 \\
\hline
\end{tabular}

Sprachkontakt wird allzu oft sogar für die Erklärung grammatischen Wandels herangezogen (dass er an lexikalischem Wandel beteiligt ist, ist unbestritten), wahrscheinlich weil eine solche Erklärung einfach und auch für Laien intuitiv zugänglich ist und weil man immer Kontaktszenarien findet, wenn man sich Mühe gibt. So wird der lange Zeit favorisierte und in vielen Einführungen immer noch nachzulesende Einfluss der lateinischen auf die deutsche Syntax nach und nach überzeugend zurückgewiesen. Dies dürfte weitgehend auch für unser Anredesystem gelten, wenngleich eine zumindest katalysatorische Wirkung des romanischen auf das deutsche System nicht auszuschließen ist. Mit dem typologischen Blick macht Simon (2003a) jedoch plausibel, dass die formal pluralische Anrede an Einzelpersonen auch ohne romanischen Kontakteinfluss entstanden sein kann: „[D]ie höfliche Pluralanrede [ist] innerhalb der Sprachen der Welt dermaßen weit verbreitet, dass unmöglich in allen Fällen von einer Beeinflussung durch das Alte Rom ausgegangen werden kann" (104).

Besonders die weiteren Ausbaustufen (er/sie im Sg. und Sie im Pl.), bei denen romanischer Kontakteinfluss versagt, legen es nahe, dass es sich um eigenständige und typologisch gut beobachtbare Entwicklungen handelt, die genau diese Implikationskette voraussetzen. Sie basieren auf dem bekannten face- bzw. Höflichkeitsmodell von Brown/Levinson (1987), das 
zwischen einem a) positive face und einem b) negative face unterscheidet: a) positive face: Das Gegenüber wird erhöht, erfährt Lob, Bestätigung und offene Wertschätzung (vgl. Allerdurchlauchtigster Fürst!), was sich auch in der Pluralisierung seiner Anrede manifestieren kann, d. h. die Wichtigkeit der Person wird durch ihre Vervielfachung ikonisiert; b) negative face: Der Adressat möchte nicht beeinträchtigt und zu nichts genötigt werden, daher lautet hier die Devise: ,give options'. Indirektheit ist hier die sprachliche Konsequenz, etwa die Imperativvermeidung bei Aufforderungen, der Gebrauch unpersönlicher Konstruktionen, häufiger Konjunktivgebrauch (ich hätte gern ein Brot - mitgedacht: wenn es Ihnen nichts ausmacht), Abschwächungen, Entschuldigungen etc. Das Ihrzen wird meist als positive Höflichkeitsstrategie interpretiert (Erhöhung der Wertigkeit des Adressaten, Machtmetapher), es kann aber auch als negative gedeutet werden, indem dem Adressaten die Option gegeben wird, „sich in eine größere Gruppe zurückzuziehen und dadurch unmittelbare Reaktionsverantwortung zu verweigern" (Simon 2003a, 105). Favorisiert man letztere Deutung, so fügt sich dies gut in das auf Indirektheit basierende, d.h. negativen Strategien folgende Gesamtbild der kategoriellen Verschiebungen (vgl. auch Head 1978): Je eher man den Blick vom Gegenüber abwendet, je weniger man es fixiert - und dies gilt gleichermaBen für sprachliche Strategien -, desto höflicher behandelt man es. Dies bedeutet: Das Gegenüber wird sprachlich möglichst diffus und vage erfasst. Die Numerusverschiebung Sg.>Pl. lässt es in einer Menschenmenge verschwinden. Was nun im 17. Jh. mit der er/sie-Anrede folgt, ist die komplette Blickabwendung, nach Simon (2003a) die „Verabwesendung“ des Gegenübers: Man tut so, als spräche man über eine dritte Person, die ja nicht einmal im Raum anwesend und damit sichtbar zu sein braucht. Der Numerusfolgt also eine sog. Personenverschiebung von 2.Person $>$ 3.Person. Im 18. Jh. steigert sich das Verfahren noch einmal, indem die Numerus- mit der Personenverschiebung kombiniert wird: Sie (mit Pl.-Kongruenz). Gleichzeitig (aber davon unabhängig) tauscht die $i h r$ - mit der er/sie-Anrede den Platz: er/sie wird zur sog. Dienstbotenanrede degradiert, während $i b r$ sich darüber setzt. Auch dies lässt sich mit dem Blickabwendungskonzept motivieren: Setzt die er/sie-Anrede immerhin die Wahrnehmung des natürlichen Geschlechts voraus, so abstrahiert die pluralische Sie-Anrede davon, d. h. das Gegenüber wird immer vager, es verschwindet immer mehr. Somit ist die Entwicklung des deutschen Anredesystems ein Paradebeispiel für die sukzessive zunehmende Indirektheit, basierend auf dem Konzept der negativen Höflichkeit. Eine andere Reihenfolge als die eingetretene ist nicht denkbar. Indem das Deutsche - trotz vielfachen Sprachkontakts - diesen eigenständigen Weg gewählt hat - es gibt nicht viele Sprachen, die so weit gegangen sind -, bedarf auch die Begründung der ersten Ausbaustufe der ihr-Anrede 
nicht zwingend des Sprachkontakts (für weitere Argumente dagegen vgl. Simon 2003a).

Für viele weitere, faszinierende Beobachtungen, auch zu anderen Sprachen und Kulturen, sowie zu neuen Perspektiven und Erklärungen hinsichtlich der deutschen Sprachgeschichte ist die genannte Monografie dringend zu empfehlen: Ohne diesen typologischen Blick über den Tellerrand bliebe unser Wissen beschränkt.

\section{Fazit und Ausblick}

Die drei Beispiele aus der historischen Phonologie, Morphologie und Pragmatik haben gezeigt, dass man sich neuer Erkenntnis- und Erklärungsmöglichkeiten beraubt, wenn man den Blick nicht über den Tellerrand der eigenen Sprach(geschicht)e richtet. Schon der Vergleich dicht verwandter Sprachen und Sprachgeschichten wie der germanischen erweist schnell, dass es viele Möglichkeiten gibt, bei ähnlichen Voraussetzungen unterschiedliche Wege einzuschlagen, und dass dies nicht immer nur dem Sprachkontakt geschuldet sein kann. So haben sich die nordgermanischen Sprachen Schwedisch und Norwegisch silbensprachlich organisiert, während Englisch, Dänisch und Deutsch wortsprachlich ausgebaut haben - mit jeweils unterschiedlichen Strategien, aber - aus typologischer Perspektive - in die gleiche Richtung gehend. Es wird der Forschung der nächsten Jahre vorbehalten sein, die vielen Strategien wortsprachlichen Ausbaus zu dokumentieren und systematisieren. Auch aus verbalmorphologischer Perspektive haben die germanischen Sprachen zwar unterschiedliche Systeme ausgebildet, doch folgen sie - mit jeweils eigenen konkreten Umsetzungen - dem typologisch gewonnenen Relevanzprinzip. So kultiviert $z$. B. das schwedische Verbalsystem einen anderen Ausschnitt aus der Relevanzskala, indem es die minderrelevanten Kategorien Person und Numerus, ja sogar Modus komplett abgebaut, doch den (hochrelevanten) Aspekt - neben dem Tempus - ausgebaut hat (hierzu vgl. Schmuck 2010). Auch die sehr verschiedenen Adressatensysteme in der Germania wären es wert, in ihrer Genese typologisch verglichen zu werden; selbstverständlich sind Dialekte gleichermaßen einzubeziehen (vgl. zum Bairischen und Niederdt. Simon 2003a, zum Afrikaans Simon 2010).

Wichtig ist bei alledem: Es geht nicht um die Erfüllung typologischer Muster um ihrer selbst willen, und noch weniger geht es um die Bestückung abstrakter Theorien durch die selektive Auswahl historischer Daten. Ausgangspunkt sind einzig die sprachhistorischen Daten und Befunde. Letztlich sind es universell geltende kognitive, aber auch anthropologische und soziale Faktoren, die für die mehr oder weniger starke Verbreitung 
sprachlicher Erscheinungen in den Sprachen der Welt verantwortlich sind. Auch hierzu erfolgt derzeit viel Wissenszuwachs, der von der Historischen Sprachwissenschaft zur Kenntnis genommen werden sollte - so wie umgekehrt die Historische Sprachwissenschaft zu dieser Diskussion beitragen kann, indem sie wertvolle Daten und Befunde zum Wandel liefert. Umso bedauerlicher ist es, dass selbst jüngst erschienene Einführungen in die deutsche Sprachgeschichte (z. B. Besch/Wolf 2009, Schmid 2009) die typologische Forschung ignorieren. Man sollte sie zumindest als alternative Erklärungsangebote neben andere stellen und es den Lesern selbst überlassen, was sie für plausibel halten. Zumindest sollte man ihnen den Weg in diese Richtung, die weit mehr verspricht als nur nach Sprachkontaktszenarien oder dem Einfluss gesellschaftlicher Veränderungen auf die Sprache zu suchen, nicht verbauen.

\section{Literatur}

Auer, Peter (2001): Silben- und akzentzählende Sprachen. In: Haspelmath, Martin et al. (eds.): Language Typology and Language Universals. An International Handbook. Vol. 2. Berlin/New York: de Gruyter, 13911399.

Augst, Gerhard (1975): Wie stark sind die starken Verben? Überlegungen zur Subklassifizierung der nhd. Verben. In: Augst, Gerhard (Hrsg.): Untersuchungen zum Morpheminventar der deutschen Gegenwartssprache. Tübingen: Narr, 231-281.

Aronoff, Mark/Fuhrhop, Nanna (2002): Restricting suffix combinations in German and English: Closing suffixes and the monosuffix constraint. In: Natural Language \& Linguistic Theory 20, 451-490.

Besch, Werner (1996): Duzen, Siezen, Titulieren. Zur Anrede im Deutschen gestern und heute. Göttingen: Vandenhoeck \& Ruprecht.

Besch, Werner (2003): Anredeformen des Deutschen im geschichtlichen Wandel. In: Besch, Werner et al. (Hrsg.): Sprachgeschichte. Ein Handbuch zur Geschichte der deutschen Sprache und ihrer Erforschung. Bd. 3. Berlin/New York: de Gruyter, 2599-2628.

Besch, Werner/Wolf, Norbert Richard (2009): Geschichte der deutschen Sprache. Längsschnitte, Zeitstufen, linguistische Studien. Berlin: Erich Schmidt.

Brown, Penelope/Levinson, Stephen (1987): Politeness: Some Universals in Language Use. Cambridge: Cambridge University Press.

Busch, Albert/Stenschke, Oliver (2007): Germanistische Linguistik. Tübingen: Narr. 
Bybee, Joan (1985): Morphology: A Study of the Relation between Meaning and Form. Amsterdam: Benjamins.

Bybee, Joan (1994): Morphological universals and change. In: Asher, Ronald E. (ed.): The Encyclopedia of Language and Linguistics. Vol. 5. Oxford: Pergamon, 2557-2562.

Bybee, Joan et al. (1994): The Evolution of Grammar: Tense, Aspect, and Modality in the Languages of the World. Chicago: Chicago University Press.

Croft, William (2003): Typology and Universals. 2nd edition. Cambridge: Cambridge University Press.

Dammel, Antje (2008): Pragmatischer Wandel. In: Nübling, Damaris et al. (2008): Historische Sprachwissenschaft des Deutschen. Eine Einführung in die Prinzipien des Sprachwandels. Tübingen: Narr, 152-173.

Fuhrhop, Nanna (1996): Fugenelemente. In: Lang, Ewald/Zifonun, Gisela (Hrsg.): Deutsch - typologisch. Berlin/New York: de Gruyter, 525-550.

Fuhrhop, Nanna (1998): Grenzfälle morphologischer Einheiten. Tübingen: Stauffenburg.

Head, Brian (1978): Respect degrees in pronominal reference. In: Greenberg, Joseph (ed.): Universals of Human Language. Vol. 3: Word Structure. Stanford: Stanford University Press, 151-211.

Henzen, Walter (1965): Deutsche Wortbildung. Tübingen: Niemeyer.

Ineichen, Gustav (1991): Allgemeine Sprachtypologie. Ansätze und Methoden. Darmstadt: Wiss. Buchgesellschaft.

Klein, Wolf Peter (2003): Sprachliche Zweifelsfälle als linguistischer Gegenstand. Zur Einführung in ein vergessenes Thema der Sprachwissenschaft. In: Linguistik online 16.4, 1-26.

Klein, Wolf Peter (2009): Auf der Kippe? Zweifelsfälle als Herausforderung(en) für Sprachwissenschaft und Sprachnormierung. In: Konopka, Marek/Strecker, Bruno (Hrsg.): Deutsche Grammatik - Regeln, Normen, Sprachgebrauch. IDS Jahrbuch 2008. Berlin, New York, 141-165.

Kürschner, Sebastian (2003): Fugenelemente im Deutschen und Dänischen - eine kontrastive Studie zu einem Grenzfall der Morphologie. Freiburg. www.freidok.uni-freiburg.de/volltexte/1256/.

Nübling, Damaris (1998): Wie die Alten sungen ... Zur Rolle von Frequenz und Allomorphie beim präteritalen Numerusausgleich im Frühneuhochdeutschen. In: Zeitschrift für Sprachwissenschaft 17.2, 185-203.

Nübling, Damaris (2000): Prinzipien der Irregularisierung. Eine kontrastive Untersuchung von zehn Verben in zehn germanischen Sprachen. Tübingen: Niemeyer.

Nübling, Damaris/Dammel, Antje (2004): Relevanzgesteuerter morphologischer Umbau im Frühneuhochdeutschen. In: Beiträge zur Geschichte der deutschen Sprache und Literatur 126.2, 177-207. 
Nübling, Damaris/Szczepaniak, Renata (2008): On the way from morphology to phonology: German linking elements and the role of the phonological word. In: Morphology 18, 1-25.

Nübling, Damaris/Szczepaniak, Renata (2009): Religion $+s+$ freibeit, Stabilität $+s+$ pakt und Subjekt $(+s+)$ pronomen: Fugenelemente als Marker phonologischer Wortgrenzen. In: Müller, Peter Otto (Hrsg.): Studien zur Fremdwortbildung. Hildesheim: Olms, 195-222.

Ortner, Lorelies et al. (1991): Deutsche Wortbildung. Typen und Tendenzen in der Gegenwartssprache. Bd. 4: Substantivkomposita. Berlin/New York: de Gruyter.

Schmid, Hans Ulrich (2009): Einführung in die deutsche Sprachgeschichte. Stuttgart/Weimar: Metzler.

Schmuck, Mirjam (2010): Relevanzgesteuerter verbalmorphologischer Wandel im Deutschen und Schwedischen. In: Dammel, Antje et al. (Hrsg.): Kontrastive Germanistische Linguistik. Hildesheim: Olms, 523-552.

Simon, Horst (1997): Die Diachronie der deutschen Anredepronomina aus Sicht der Universalienforschung. In: Sprachtypologie und Universalienforschung 50.3, 267-281.

Simon, Horst (2003a): Für eine grammatische Kategorie ,Respekt ${ }^{`}$ im Deutschen. Synchronie, Diachronie und Typologie der deutschen Anredepronomina. Tübingen: Niemeyer.

Simon, Horst (2003b): From pragmatics to grammar: Tracing the development of respect in the history of the German pronouns of address. In: Taavitsainen, Irma/Jucker, Andreas H. (eds.): Diachronic Perspectives on Address Term Systems. Amsterdam/Philadelphia: Benjamins, 85123.

Simon, Horst (2010): Zur Grammatik der Anrede im Afrikaans und im älteren Deutsch. In: Dammel, Antje et al. (Hrsg.): Kontrastive Germanistische Linguistik. Hildesheim: Olms, 395-423.

Szczepaniak, Renata (2007): Der phonologisch-typologische Wandel des Deutschen von einer Silben- zu einer Wortsprache. Berlin/New York: de Gruyter.

Szczepaniak, Renata (2008): Phonologischer Wandel. In: Nübling, Damaris et al. (2008): Historische Sprachwissenschaft des Deutschen. Eine Einführung in die Prinzipien des Sprachwandels. Tübingen: Narr, 11-42.

Tomczyk-Popińska, Ewa (1987): Linguistische Merkmale der deutschen gesprochenen Standardsprache. In: Deutsche Sprache 15, 336-357.

Wegener, Heide (2006): Statistical evidence for the role of phonology in the distribution and motivation of the linking element $-s$ - in German. In: Pre-proceedings of the International Conference on Linguistic Evidence, Empirical, Theoretical and Computational Perspectives, Tübingen, 2-4 February 2006. Tübingen: SFB 441, 201-203. 